\title{
Contents
}

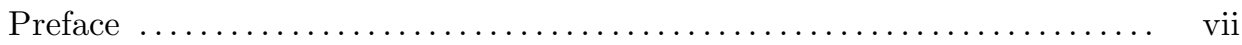

Scientific Portrait of

Alexander Vasilievich Kazhikhov ............................ ix

G.V. Alekseev and D.A. Tereshko

Boundary Control Problems for Stationary Equations

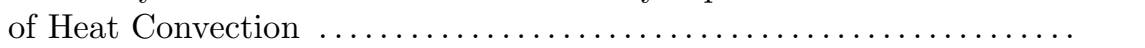

$Y$. Amirat and V. Shelukhin

Homogenization of the Poisson-Boltzmann Equation $\ldots \ldots \ldots \ldots \ldots \ldots 23$

S.N. Antontsev and N.V. Chemetov

Superconducting Vortices: Chapman Full Model ............... 41

D. Bresch, E.D. Fernández-Nieto, I.R. Ionescu and P. Vigneaux

Augmented Lagrangian Method and Compressible Visco-plastic

Flows: Applications to Shallow Dense Avalanches ............... 57

D. Bresch, B. Desjardins and E. Grenier

Oscillatory Limits with Changing Eigenvalues: A Formal Study .... 91

A.Yu. Chebotarev

Finite-dimensional Control for the Navier-Stokes Equations . . . . . . . 105

H. Beirão da Veiga

On the Sharp Vanishing Viscosity Limit

of Viscous Incompressible Fluid Flows $\ldots \ldots \ldots \ldots \ldots \ldots \ldots \ldots \ldots$

E. Feireisl and A. Novotný

Small Péclet Number Approximation as a Singular Limit

of the Full Navier-Stokes-Fourier System with Radiation

E. Feireisl and A. Vasseur

New Perspectives in Fluid Dynamics: Mathematical Analysis

of a Model Proposed by Howard Brenner 
J. Frehse and M. Růżička

Existence of a Regular Periodic Solution to the

Rothe Approximation of the Navier-Stokes Equation

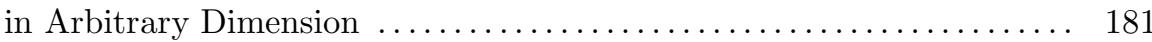

A.V. Fursikov and R. Rannacher

Optimal Neumann Control for the Two-dimensional

Steady-state Navier-Stokes equations $\ldots . \ldots \ldots \ldots \ldots \ldots \ldots \ldots \ldots \ldots . . \ldots \ldots$

S. Itoh, N. Tanaka and A. Tani

On Some Boundary Value Problem for the Stokes Equations

with a Parameter in an Infinite Sector ........................

A. Khludnev

Unilateral Contact Problems Between an Elastic Plate

W. Layton and A. Novotný

On Lighthill's Acoustic Analogy for Low Mach Number Flows ...... 247

A.E. Mamontov

On the Uniqueness of Solutions to Boundary Value Problems

for Non-stationary Euler Equations

M. Padula

On Nonlinear Stability of MHD Equilibrium Figures

V.V. Pukhnachev

Viscous Flows in Domains with a Multiply Connected Boundary .....

E.V. Radkevich

Problems with Insufficient Information about

Initial-boundary Data

\section{V.A. Solonnikov}

On the Stability of Non-symmetric Equilibrium Figures

of Rotating Self-gravitating Liquid not Subjected

to Capillary Forces

V.N. Starovoitov and B.N. Starovoitova

Dynamics of a Non-fixed Elastic Body 